\title{
Tissue transglutaminase triggers oligomerization and activation of dual leucine zipper-bearing kinase in calphostin C-treated cells to facilitate apoptosis
}

\author{
K Robitaille ${ }^{1}$, A Daviau', J Tucholski ${ }^{2}$, GVW Johnson ${ }^{2}$, \\ C Rancourt ${ }^{3}$ and R Blouin ${ }^{\star, 1}$ \\ ${ }^{1}$ Département de biologie, Faculté des sciences, Université de Sherbrooke, \\ Sherbrooke, Québec J1K 2R1, Canada \\ 2 Department of Psychiatry and Behavioral Neurobiology, University of Alabama \\ at Birmingham, Birmingham, AL, USA \\ ${ }^{3}$ Département de microbiologie, Faculté de médecine, Université de \\ Sherbrooke, Sherbrooke, Québec J1H 5N4, Canada \\ * Corresponding author: R Blouin, Département de biologie, Faculté des \\ sciences, Université de Sherbrooke, 2500, boulevard de l'Université, \\ Sherbrooke (Québec), Canada J1K 2R1. Tel: + 8198218000 ext. 2062; \\ Fax: + 819821 8049; E-mail: Richard.Blouin@USherbrooke.ca
}

Received 29.10.03; accepted 03.12.03; published online 23.1.04 Edited by M Piacentini

\begin{abstract}
Although tissue transglutaminase (tTG) has been recognized as a mediator of apoptosis in various experimental models, little is currently known about the molecular mechanisms by which this protein modulates cell death. Recent work from our laboratory has shown that activation of tTG in cells exposed to the apoptotic inducer calphostin $\mathrm{C}$ triggers the crosslinking of dual leucine zipper-bearing kinase (DLK), a proapoptotic kinase acting as an essential component of the C-Jun aminoterminal kinase (JNK) signaling pathway. As a consequence of this observation, we have undertaken experiments to investigate the functional relevance of DLK oligomerization in tTG-mediated apoptosis. Our results indicate that, in cells undergoing calphostin C-induced apoptosis, tTG-dependent DLK oligomerization occurs early in the apoptotic response. Both immunocomplex kinase assays and immunoblotting with phosphospecific antibodies revealed that oligomer formation by tTG-mediated crosslinking reactions significantly enhanced the kinase activity of DLK and its ability to activate the JNK pathway. Moreover, functional studies demonstrate that ITG-mediated oligomerization of wild-type DLK sensitizes cells to calphostin C-induced apoptosis, while crosslinking of a kinase-inactive variant of DLK does not. Collectively, these data strongly suggest that tTG facilitates apoptosis, at least partly, by oligomerization and activation of the proapoptotic kinase DLK.

Cell Death and Differentiation (2004) 11, 542-549.

doi:10.1038/sj.cdd. 4401392

Published online 23 January 2004
\end{abstract}

Keywords: tissue transglutaminase; dual leucine zipper-bearing kinase; apoptosis; calphostin C; c-Jun amino-terminal kinase; oligomerization

\begin{abstract}
Abbreviations: tTG, tissue transglutaminase; DLK, dual leucine zipper-bearing kinase; JNK, c-Jun N-terminal kinase; MAPK, mitogen-activated protein kinase; MDC, monodansylcadaverine; SDS-PAGE, sodium dodecyl sulfate-polyacrylamide gel electrophoresis; GST, glutathione $S$-transferase
\end{abstract}

\section{Introduction}

Tissue transglutaminase (tTG, also called transglutaminase II) ${ }^{1}$ is a ubiquitously expressed bifunctional enzyme that catalyzes in $\mathrm{C} \mathrm{Ca}^{2+}$-dependent manner the transamidation of selected peptide-bound glutamine residues in target proteins. ${ }^{1-3}$ This activity causes either the formation of covalent bonds within or between polypeptide chains, or the incorporation of polyamines into substrate proteins. ${ }^{1-3}$ Such transglutaminase-mediated crosslinking or polyamination of substrate proteins have been proposed to regulate important biological processes, including cell proliferation, differentiation, and wound healing. ${ }^{1-3}$ Besides acting as a transamidating enzyme, tTG is also known to bind and hydrolyze GTP and ATP in a $\mathrm{Ca}^{2+}$-independent manner. ${ }^{4,5}$ Consistent with this activity, it was reported that $\mathrm{TTG}$ can function as a $\mathrm{G}$ protein in transmembrane signaling from activated serpentine receptors to phospholipase $\mathrm{C} \delta$, an enzyme responsible for the production of the second messengers inositol-1,4,5-triphosphate and diacylglycerol. ${ }^{6,7}$

Many independent laboratories have identified tTG as a potentially important regulator of apoptosis in various experimental models. However, since both proapoptotic and antiapoptotic activities have been reported in the literature, its exact role in programmed cell death has not been clearly delineated so far. Experimental evidence supporting a proapoptotic role derives from studies demonstrating that expression and activity of tTG are both substantially increased at the onset of apoptosis in numerous cell types. ${ }^{8-10}$ Accordingly, transfection-based experiments showed that expression of tTG alone primes cells for spontaneous and stimuli-induced apoptosis, ${ }^{11-13}$ whereas inhibition of its expression with antisense constructs significantly decreases cell death. ${ }^{14}$ On the other hand, recent reports also suggest that ITG has antiapoptotic effects under certain conditions. For instance, upregulation of tTG expression by retinoic acid (RA) has been shown to protect human HL60 cells and mouse NIH 3T3 fibroblasts against apoptosis induced by treatment with a synthetic retinoid, $\mathrm{N}$-(4-hydroxyphenyl)retinamide (HRP). ${ }^{15}$ Other studies have also indicated that inhibition of tTG activity with monodansylcadaverine (MDC) significantly reduces the viability of NIH 3T3 cells exposed to RA. ${ }^{15,16}$

The mechanisms that may account for the antiapoptotic and proapoptotic properties of tTG within the cell are still largely misunderstood. Nevertheless, it is possible that the dual activity of tTG in apoptosis may be linked to its ability to 
transamidate specific cellular components that are thought to be involved in the regulation of the apoptotic machinery. Interestingly, one molecule that has been found to play an important role in the antiapoptotic activity of tTG is the retinoblastoma protein $(\mathrm{pRb})$, a regulator of cell division, differentiation, and survival. ${ }^{17}$ Indeed, $\mathrm{pRb}$ has been reported to be a substrate of tTG in cells undergoing calphostin Cinduced apoptosis, ${ }^{18}$ and its transamidation in vitro provides protection against caspase-mediated degradation. ${ }^{19}$ In addition, using fibroblasts derived from $\mathrm{pRb}$-deficient mice, it has been demonstrated that $\mathrm{TTG}$ requires the presence of $\mathrm{pRb}$ to exhibit antiapoptotic activity. ${ }^{19}$ Like $p R b,{ }^{18}$ dual leucine zipper-bearing kinase (DLK) is another molecule that has been found to undergo tTG-dependent oligomerization in cells exposed to calphostin $\mathrm{C} .{ }^{20}$ This protein belongs to a subgroup of serine/threonine protein kinases, referred to as the mixedlineage kinases (MLKs), that act as key regulators of the stress-activated c-Jun N-terminal kinase (JNK) mitogenactivated protein kinase (MAPK) signaling pathway. ${ }^{21}$ Consistent with its function as an inducer of JNK activity, DLK is also known to contribute to apoptosis in neuronal cells. ${ }^{22}$ Indeed, overexpression of DLK in PC12 cells and in sympathetic neurons was shown to induce apoptosis via the mitochondrial- and caspase-dependent death pathway. ${ }^{22}$ Conversely, overexpression of a dominant-negative form of DLK in these cells blocked apoptosis caused by nerve growth factor withdrawal or by ectopic expression of activated forms of the small GTPases Rac1 and Cdc42. Together, these studies indicate that DLK is implicated in the control of cell death. Whether crosslinking of DLK by tTG might function to modulate apoptosis in cells exposed to calphostin $\mathrm{C}$ is not clear.

Given that DLK is an essential component of the JNK MAPK pathway, which regulates the apoptotic machinery in mammalian cells, ${ }^{23-25}$ we addressed the possibility that its polymerization in cells might represent an effective regulatory mechanism by which tTG modulates apoptosis. Our results show that activation of TTG in calphostin $\mathrm{C}$-treated cells results in the polymerization of DLK during the early phases of the apoptotic response, and that this event increases the catalytic activity of DLK as well as its ability to stimulate the JNK pathway. Moreover, we demonstrate that tTG-mediated oligomerization/activation of DLK has a priming effect on apoptosis induced by calphostin C, suggesting that the crosslinked/activated form of DLK contributes to the proapoptotic activity of tTG.

\section{Results}

\section{Effects of calphostin C on in situ tTG activity and DLK status}

Calphostin C, a potent inhibitor of protein kinase $C$, induces apoptosis in a variety of cellular backgrounds by an unknown mechanism. ${ }^{26,27}$ Since recent studies have shown that calphostin $\mathrm{C}$ stimulates the transamidating activity of tTG in a time-dependent manner, ${ }^{18,33}$ we began this study by monitoring the in situ transamidating activity of tTG in mouse NIH 3T3 fibroblasts that were exposed to calphostin C for various lengths of time (Figure 1a). In agreement with the

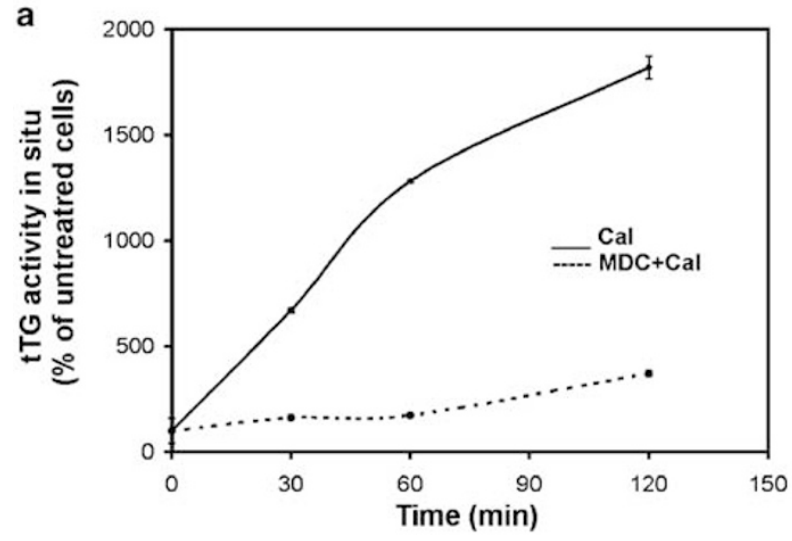

b

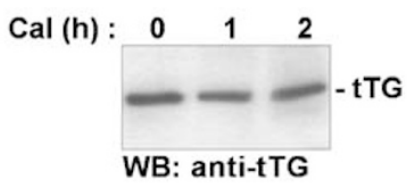

C

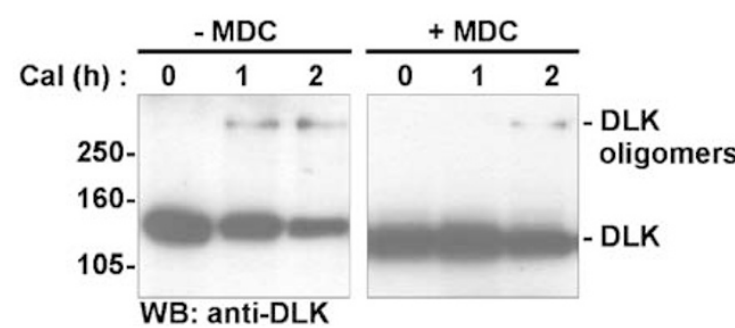

Figure 1 Production of tTG-dependent DLK oligomers in calphostin C-treated $\mathrm{NIH} 3 \mathrm{~T} 3$ cells. (a) Treatment of NIH 3T3 cells with $250 \mathrm{nM}$ calphostin C (Cal) resulted in a significant time-dependent increase in in situ ITG activity (-). Incubation of cells with the TTG inhibitor MDC $(0.5 \mathrm{mM})$ for $1 \mathrm{~h}$ prior to treatment with calphostin C (Cal) resulted in inhibition of in situ tTG activity (- - - -). Data are presented as a percentage of the activity in the absence of calphostin $\mathrm{C}$. (b) Cultures of NIH 3T3 cells were incubated with $250 \mathrm{nM}$ calphostin C (Cal) for the indicated time periods, lysed, and subjected to immunoblotting analysis with an antibody against tTG. (c) Cultures of $\mathrm{NIH} 3 \mathrm{~T} 3$ cells were incubated in the absence $(-)$ or presence $(+)$ of MDC $(0.5 \mathrm{mM})$ for $1 \mathrm{~h}$ prior to treatment with $250 \mathrm{nM}$ calphostin $\mathrm{C}$ (Cal) for the indicated time periods. After incubation, cells were lysed and subjected to immunoblotting analysis with a DLK antiserum. The data in this figure and all the other figures are a representative of at least three independent experiments

previous findings, ${ }^{18,28}$ we detected a gradual and dramatic increase in tTG activity as early as $30 \mathrm{~min}$ following treatment with $250 \mathrm{nM}$ calphostin $\mathrm{C}$, a dose known to decrease survival in several cell types. ${ }^{29,30}$ Immunoblots processed in parallel with lysates prepared from these cells and an antibody specifically targeted to $\mathrm{TTG}$ confirmed that the increase in transamidating activity was not attributable to variations in tTG protein levels (Figure 1b). Calphostin C exposure, therefore, appears to be able to stimulate the transamidating activity of tTG in NIH 3T3 cells.

Calphostin C-induced activation of tTG in NIH 3T3 cells was also found to be paralleled by the formation of high molecular weight DLK oligomers (greater than $250 \mathrm{kDa}$ in apparent mobility), which were readily detectable just below the junction of the stacking and running gels (Figure 1c). To confirm that 
calphostin C-induced oligomerization of DLK was indeed attributable to tTG activation, we next performed immunoblotting experiments with NIH 3T3 cells that had been pretreated with MDC, a specific inhibitor of transglutaminase activity, ${ }^{31}$ prior to calphostin $\mathrm{C}$ exposure. As shown in Figure 1 ( $a$ and $c$ ), MDC almost completely antagonized tTG activation as well as the formation of DLK oligomers in calphostin C-treated cells. These findings, therefore, led us to suggest that accumulation of DLK oligomers in response to calphostin $\mathrm{C}$ is mediated by tTG.

\section{tTG-mediated polymerization of DLK in calphostin C-treated cells precedes poly-ADP-ribose polymerase (PARP) cleavage}

To investigate the relative contribution of tTG-catalyzed DLK polymerization during apoptosis, we first examined the position of endogenous DLK oligomerization in the cascade of events leading to the establishment of the 'death' phenotype. For this purpose, NIH 3T3 cells were exposed to calphostin $\mathrm{C}$ for various times, lysed, and subjected to immunoblot analyses with a specific antibody to DLK (Figure 2a). In this instance, we note the appearance of DLK oligomers as early as $15 \mathrm{~min}$ after exposure to calphostin $\mathrm{C}$ with a gradual progression toward maximal levels at $45 \mathrm{~min}$. Concurrent immunoblotting of the lysates with an antibody specific for the cleaved form of PARP, a caspase substrate, ${ }^{32}$ revealed that calphostin $\mathrm{C}$ was able to stimulate PARP cleavage approximately $75 \mathrm{~min}$ after initiation of the treatment. Taken together, these results demonstrate that DLK oligomerization in calphostin C-treated NIH 3T3 cells is encountered during the early phases of the apoptotic response, namely during the time period preceding PARP cleavage.

In conjunction with these experiments, we also investigated whether calphostin C-induced DLK oligomerization and PARP cleavage both required transglutaminase activity. NIH 3T3 cells were preincubated with MDC for $1 \mathrm{~h}$ before being exposed to calphostin $C$ and processed for immunoblot analyses. As shown in Figure $2 b$, treatment with MDC almost completely prevented the calphostin C-mediated accumulation of DLK oligomers and subsequent cleavage of PARP, thereby suggesting that tTG contributes to DLK oligomerization and apoptosis in response to this cell death stimulus.

\section{Calphostin $C$ induces oligomerization and activation of DLK in a tTG-dependent manner}

As is the case for several members of the protein kinase family, dimerization or oligomerization is thought to contribute to the activation of DLK. ${ }^{33}$. Consequently, we tested whether the catalytic activity of DLK and its ability to activate JNK were modulated in response to calphostin C-induced oligomerization. Cultures of COS-7 cells transiently transfected with an expression vector encoding a T7 epitope-tagged DLK were treated with $250 \mathrm{nM}$ calphostin $\mathrm{C}$ for 30,60 , or 120 min, lysed, and the extracts were subjected to immunoblotting with the T7 antibody and to a DLK immunocomplex kinase assay using myelin basic protein (MBP) as a substrate. As expected a
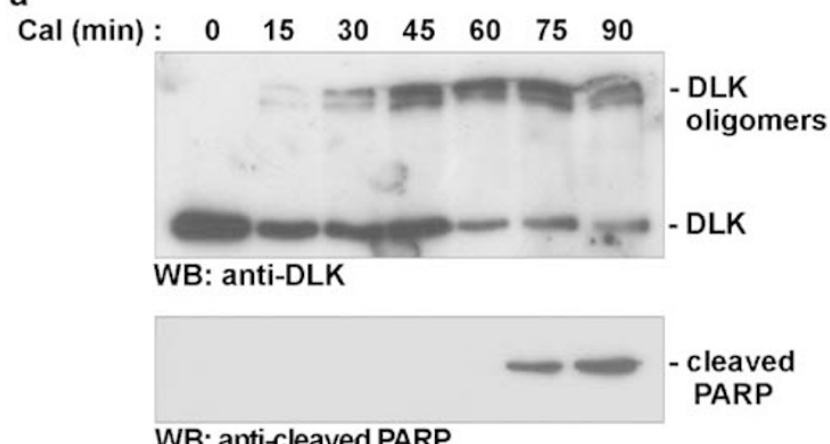

WB: anti-cleaved PARP

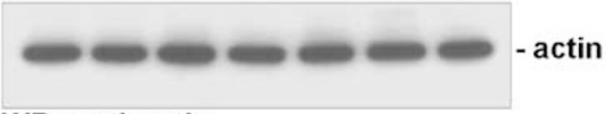

WB: anti-actin

b

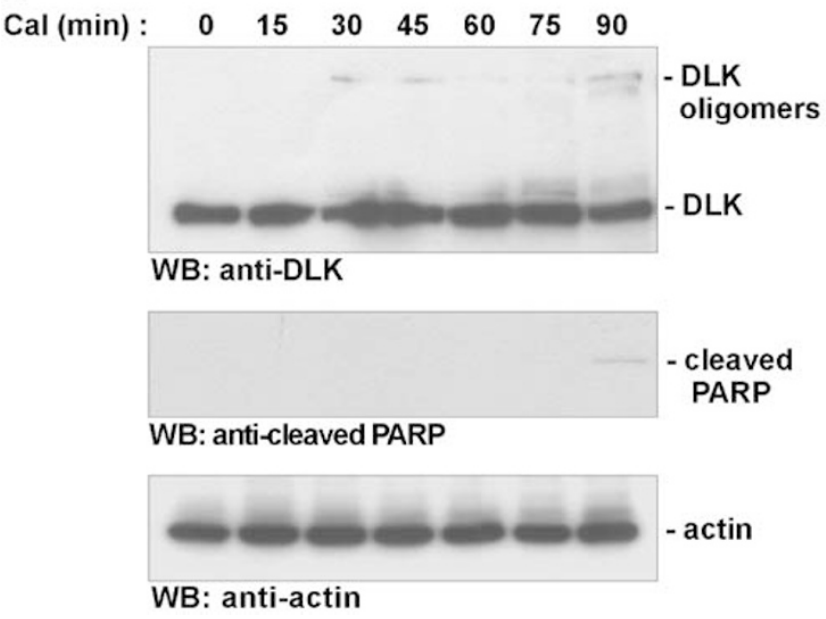

Figure 2 tTG-dependent oligomerization of DLK in the early phases of calphostin C-induced apoptosis. (a) Cultures of NIH $3 \mathrm{~T} 3$ cells were incubated with $250 \mathrm{nM}$ calphostin C (Cal) for the indicated time periods, lysed, and subjected to immunoblotting analyses using antibodies to DLK and cleaved PARP. (b) NIH 3T3 cells were exposed to the tTG inhibitor MDC for $1 \mathrm{~h}$ prior to treatment with $250 \mathrm{nM}$ calphostin C (Cal) for the indicated time periods. After incubation, cells were harvested, lysed, and subjected to immunoblotting with antibodies to DLK and cleaved PARP. As a control for protein loading, immunoblots were probed in parallel with an antibody targeting $\gamma$-actin

(Figure 3a), calphostin C treatment of transfected COS cells resulted in the progressive accumulation of the high molecular weight forms of DLK. Interestingly, the generation of these high molecular weight complexes in transfected cells was paralleled by a significant increase in the catalytic activity of DLK (Figure 3a). Concurrent immunoblotting of the cellular extracts with an antibody specific to phospho$\left(p T^{183}{ }^{18 T y r}{ }^{185}\right)$-JNK, an indicator of JNK activation, revealed that DLK oligomerization in calphostin C-treated cells was also accompanied by a gradual increase in JNK phosphorylation, the maximal level being attained when DLK was almost completely oligomerized. In comparison, calphostin $\mathrm{C}$ treatment of cells transfected with an empty 
vector had little effect on the phosphorylation of JNK. The results of these experiments demonstrate that DLK becomes activated upon calphostin $\mathrm{C}$-induced oligomerization and that such modification causes JNK activation.

a

\begin{tabular}{lllllllll} 
Cal (min): & \multicolumn{3}{c}{ Empty vector } & & \multicolumn{3}{c}{ T7-DLK } \\
\cline { 2 - 4 } \cline { 5 - 8 } & 30 & 60 & 120 & & 0 & 30 & 60 & 120
\end{tabular}

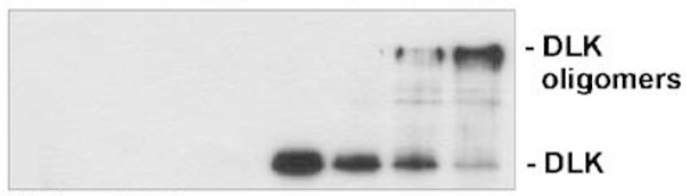

WB: anti-T7

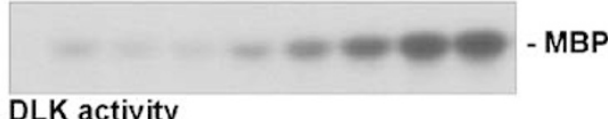

DLK activity
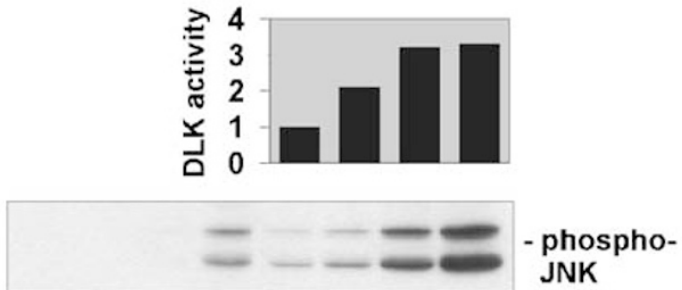

WB: anti-phosphoJNK

JNK

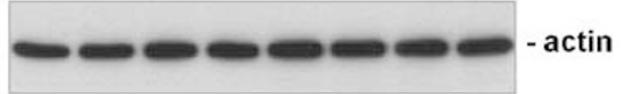

WB: anti-actin

b

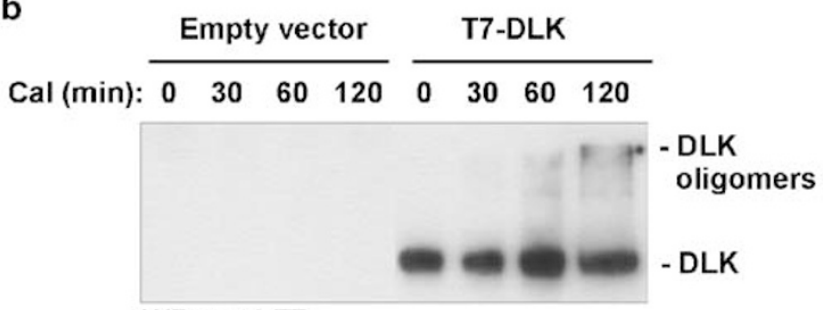

WB: anti-T7

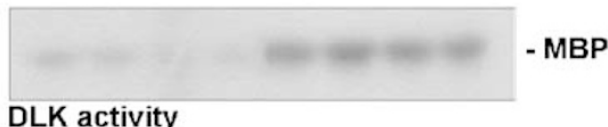

DLK activity
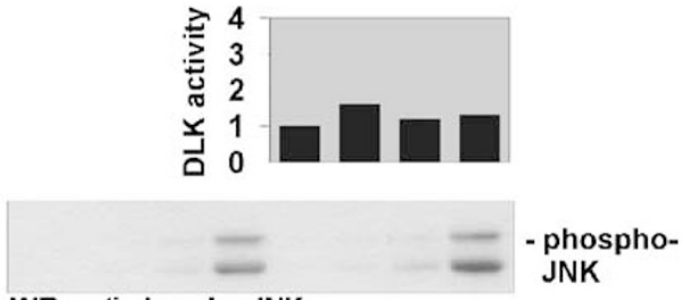

WB: anti-phospho-JNK

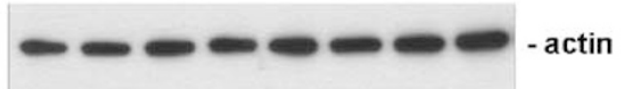

WB: anti-actin
In view of the results described above, we next examined whether calphostin $\mathrm{C}$-induced oligomerization and activation of DLK were transglutaminase dependent. COS-7 cells transiently transfected with the T7 epitope-tagged DLK expression construct were preincubated with MDC for $1 \mathrm{~h}$ before supplementing the culture medium with calphostin $C$, lysed, and the extracts analyzed by immunobloting and DLK immunocomplex kinase assays. As shown in Figure $3 \mathrm{~b}$, treatment of transfected cells with MDC almost completely prevented the calphostin $\mathrm{C}$-induced accumulation of DLK oligomers as well as the activation of DLK, thereby suggesting that ITG is involved in the oligomerization and consequent activation of DLK in response to calphostin $C$ treatment. Western blot analyses of the cell lysates, using the antiphospho-JNK antibody, revealed that JNK activation by DLK in calphostin C-treated cells was also blocked by pretreatment with MDC. Therefore, the oligomerization of DLK and activation of JNK by calphostin C appear to be functionally linked.

\section{tTG-mediated oligomerization of DLK facilitates cell commitment to apoptosis}

The experiments described above demonstrated the existence of a strong correlation between tTG activation, DLK oligomerization, and apoptosis induction in calphostin Ctreated cells. As a logical extension of these results, we decided to directly examine whether activated tTG could modulate cell commitment to apoptosis through the polymerization and activation of DLK. To address this, SH-SY5Y neuroblastoma cells stably expressing human tTG (SH/tTG) were infected with recombinant adenoviruses expressing either wild-type DLK, kinase-defective DLK, or the control green fluorescent protein (GFP) and then exposed to calphostin $\mathrm{C}$ for various lengths of time. Oligomerization of DLK in cells infected with the recombinant adenoviruses encoding wild-type and kinase-defective DLK was confirmed by probing a Western blot of the lysates with an antibody against DLK (Figure 4). JNK activity was determined in the lysates from these cells by a solid-phase kinase assay using glutathione $S$-transferase (GST)-c-Jun(1-79) fusion protein immobilized on glutathione-sepharose beads. ${ }^{45}$ Our results using this assay indicated that JNK was activated within $15 \mathrm{~min}$ in SH/tTG cells expressing wild-type DLK, with maximum activity reached when oligomerization was almost complete. Despite its ability to oligomerize in response to calphostin $\mathrm{C}$, however, the kinase-defective DLK mutant consistently failed to stimulate the activity of JNK, indicating that this response is correlated with expression of activated

Figure 3 Calphostin C promotes oligomerization and activation of DLK in a tTG-dependent manner. (a) COS-7 cells were transiently transfected with empty expression vector or a vector encoding a T7 epitope-tagged DLK. At $48 \mathrm{~h}$ after transfection, cells were treated with $250 \mathrm{nM}$ calphostin $\mathrm{C}$ (Cal) for the time indicated. Cell lysates were processed for immunoblotting with antibodies to the T7 tag, phosphorylated JNK, or $\gamma$-actin. In parallel experiments, T7-DLK was immunoprecipitated from cellular lysates with the $T 7$ antibody and its activity was determined by in vitro kinase assay using MBP as a substrate. Densitometry analysis of MBP phosphorylation was used to determine the relative levels of DLK activation at different time points. (b) Same as (a) except that cells were exposed to the tTG inhibitor MDC for $1 \mathrm{~h}$ prior to treatment with calphostin $\mathrm{C}$ (Cal) 


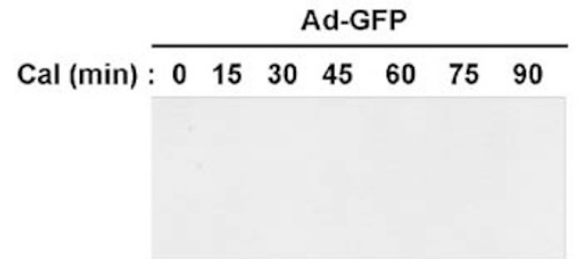

WB: anti-DLK

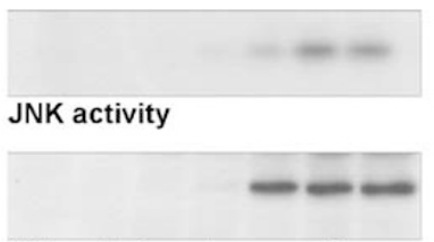

WB: anti-cleaved caspase-9

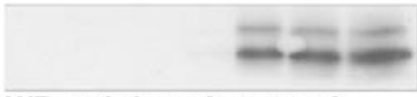

WB: anti-cleaved caspase-3
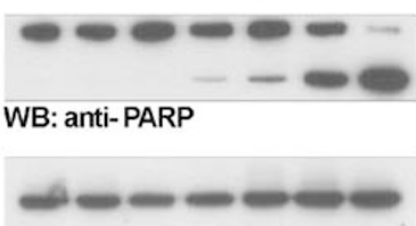

WB: anti-actin
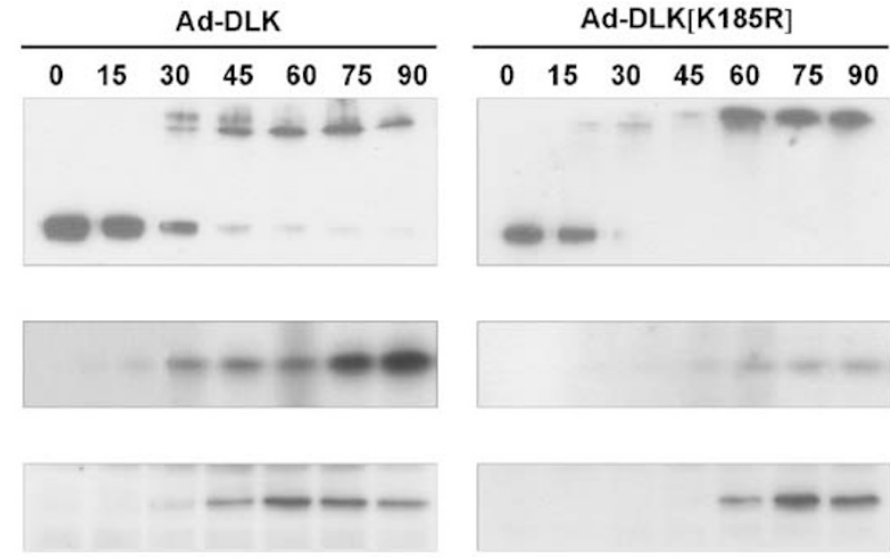

- cleaved caspase-9
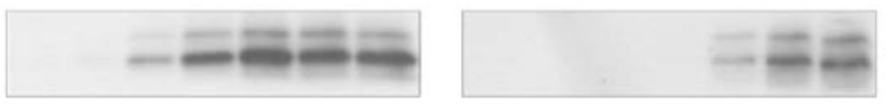

- cleaved caspase-3
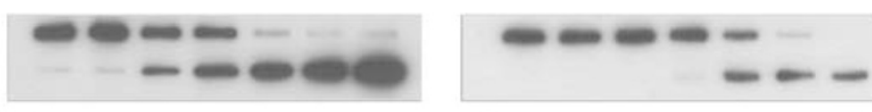

- PARP

- cleaved

PARP
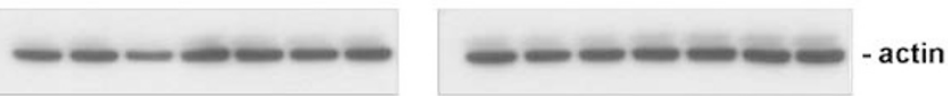

Figure 4 Oligomerization of DLK by tTG facilitates apoptosis in response to calphostin C. Human neuroblastoma SH-SY5Y cells stably expressing wild-type tTG were infected with the recombinant adenoviral vector Ad-DLK, Ad-DLK,[K185R] or the control vector Ad-GFP at a MOI of 5 for $24 \mathrm{~h}$. Cells were treated with $250 \mathrm{nM}$ calphostin $\mathrm{C}$ (Cal) for the indicated time periods, harvested, and analyzed by immunoblotting for DLK, cleaved caspase-9, cleaved caspase-3, PARP, and $\gamma$-actin. JNK activity was determined in the lysates from these cells by a solid-phase kinase assay using GST-c-Jun fusion protein immobilized on glutathione-sepharose beads

DLK. Apoptosis was assessed at different times after treatment by probing the cellular lysates with antibodies against cleaved forms of caspase- 9 and caspase- 3 , two of the key effector molecules in apoptotic cell death, and PARP. Results shown in Figure 4 reveal that SH/tTG cells, which express wild-type DLK, were much more susceptible to apoptosis induced by calphostin $\mathrm{C}$ than $\mathrm{SH} / \mathrm{tTG}$ cells infected with the adenoviruses encoding either GFP as a control or the kinase-defective mutant form of DLK. In fact, calphostin $C$ was able to stimulate the cleavage of caspase- 9 , caspase-3, and PARP as early as 30 min after initiation of the treatment in $\mathrm{SH} /$ tTG cells expressing wild-type DLK compared with 60 min for the control cells. These findings, therefore, led us to conclude that tTG-mediated oligomerization/activation of DLK has a priming effect on apoptosis induced by calphostin $\mathrm{C}$.

\section{Discussion}

tTG is a multifaceted molecule that is involved in the regulation of many cellular events, including apoptosis. However, the role of tTG in dying cells remains elusive since it can promote or prevent apoptosis, depending on the cell context and the nature of the death stimulus. Recent studies from independent laboratories have revealed that the positive or negative effects of tTG on apoptosis are modulated by both its transamidating (e.g. protein crosslinking and polyamination) and nontransamidating (e.g. GTP binding/hydrolysis) functions. ${ }^{13,15,16}$ Activation of the transamidating function of tTG in cells undergoing spontaneous or stress-induced apoptosis has been found to cause the polymerization of specific cytosolic, cytoskeletal, and nuclear substrate proteins, which are thought to contribute in some as yet unspecified way to the early and late stages of the apoptotic process. ${ }^{1,34,35}$ In this study, we demonstrate that tTG, in its protein crosslinking configuration, catalyzes the polymerization of the proapoptotic kinase DLK, and that such modification results in upregulation of DLK activity and subsequent stimulation of the JNK signaling pathway. In accord with these observations, we also provide evidence suggesting that formation of catalytically active tTG-dependent DLK oligomers facilitates cell commitment to apoptosis. These findings, therefore, lead us to propose that the proapoptotic effects of tTG can be partially attributed to its ability to increase directly the kinase activity of DLK and potentiate DLK-dependent apoptosis.

A number of recent reports have established that tTG possesses proapoptotic functions under certain conditions in diverse experimental cell models. Human neuroblastoma SK$\mathrm{N}-\mathrm{BE}^{2}$ cells overexpressing $\mathrm{tTG}$ displayed increased sensitivity to death stimuli by a mechanism that presumably involves hyperpolarization of the mitochondrial membrane. ${ }^{36}$ Similarly, overexpression of tTG in the SH-SY5Y cell line has been shown to enhance the susceptibility to apoptosis when the death stimulus promotes activation of its transamidating function. ${ }^{13}$ In accordance with these data, it was also observed in the same study that expression of a transamidation-defective mutant of tTG failed to facilitate apoptosis in 
response to stressful stimuli. Thus, the upregulation of tTG activity observed in apoptotic cells likely constitutes one of the key elements contributing to this particular type of cell death. Interestingly, our results indicate that the transamidating function of tTG can be rapidly and strongly switched on following exposure of cells to calphostin $C$, that triggers calcium-dependent apoptosis. ${ }^{27}$ One of the consequences of this activation is the formation of DLK oligomers in the early phases of the apoptotic response, namely during the time period preceding the cleavage of PARP. Inhibition of tTG by treatment with MDC blocked both DLK oligomerization and PARP cleavage, suggesting that activation of tTG is necessary to promote DLK oligomerization and to induce apoptosis in cells exposed to calphostin C. Investigation of the relationship which exists between these three events allowed us to demonstrate that oligomer formation through tTG-mediated crosslinking reactions significantly enhances the catalytic activity of DLK, as judged by the phosphorylation of MBP in immunocomplex kinase assays. In agreement with this observation, we have also shown that tTG-mediated DLK oligomerization induces the phosphorylation and activation of endogenous JNK. Taken together, these results suggest an important role for oligomerization in the control of DLK activation and consequent stimulation of the JNK pathway. Consistent with this possibility, it was reported that dimerization of monomeric DLK promotes autophosphorylation and JNK activation. ${ }^{33}$ Thus, on the basis of these data, we propose that tTG, through its crosslinking properties, functions as a positive regulator of DLK, which in turn activates the JNK signaling pathway.

According to our data, tTG-mediated oligomerization/ activation of DLK may have a significant impact on the cascade of events leading to the establishment of the programmed cell death phenotype. Indeed, our studies on the kinetics of apoptosis induction in SH/tTG cells indicate that DLK oligomerization has a priming effect on cell death initiated by calphostin $C$. This effect is entirely dependent on the kinase activity of DLK since a catalytically inactive DLK mutant consistently failed to facilitate calphostin $\mathrm{C}$-induced apoptosis in SH/tTG cells, even if its oligomerization was comparable to that of the wild-type form. Therefore, our results suggest that DLK mediates its proapoptotic activity in calphostin C-treated cells by phosphorylation and activation of downstream effectors such as JNK. In many cases, activation of JNK leads to cell signaling events that are proapoptotic. ${ }^{23-25}$ In some cell types, such as in neuronal cells, JNK has been recognized as an important regulator of cell death evoked by a variety of apoptotic stimuli, including excitotoxic stress, withdrawal of growth factors, DNA damage, oxidative stress, and $\beta$-amyloid exposure. ${ }^{37-39}$ Once activated, JNK phosphorylates and activates the transcription factor c-Jun, which is crucial for the induction of apoptosis. ${ }^{40}$ In light of these data, it is therefore possible that DLK, in its crosslinked/activated state, mediates its proapoptotic effects at least partly through activation of the JNK signaling pathway. This hypothesis is supported by the finding that dominant-negative forms of the dual specificity MAPK kinase MKK7 and the transcription factor c-Jun, two components of the JNK pathway downstream of DLK, block death caused by overexpression of DLK in neuronal PC12 cells. ${ }^{22}$
In summary, our findings indicate that DLK is an intracellular target of tTG in cells undergoing calphostin C-induced apoptosis. Upon oligomerization, DLK functions as constitutively active protein kinase, which through phosphorylation and activation of effector molecules, sensitizes cells to apoptosis. Based on these observations, we propose that modulation of DLK activity by crosslinking represents an important in vivo mechanism by which tTG regulates apoptosis.

\section{Materials and Methods}

\section{Chemicals and antibodies}

Calphostin $C$ was purchased from Calbiochem-Novabiochem Co. (San Diego, CA, USA). MDC, protease inhibitors, and all other common reagents were obtained from Sigma-Aldrich Canada Ltd. (Oakville, Ontario). BAP(5-(biotinamido)pentylamine ) and HRP-conjugated streptavidin were from Pierce Biotechnology (Rockford, IL, USA). The rabbit polyclonal antiserum to DLK was described previously. ${ }^{41}$ Rabbit polyclonal or mouse monoclonal antibodies raised against cleaved caspase-9, cleaved caspase-3, poly(ADP-ribose)polymerase (PARP), cleaved PARP, and phospho-JNK were purchased from Cell Signaling Technology Inc. (Beverly, MA, USA). The rabbit polyclonal antibody against $\gamma$-actin was obtained from Sigma-Aldrich Ltd. The mouse monoclonal antibody for the detection of the T7 epitope tag was purchased from Novagen Inc. (Madison, WI, USA). The rabbit polyclonal antibody against tTG was from Lab Vision Corp. (Fremont, CA, USA). Cell culture reagents were from Invitrogen Corp. (Carlsbad, CA, USA), HyClone Laboratories (Logan, UT, USA), Cambrex Corp. (East Rutherford, NJ, USA) or Biosource International Inc. (Camarillo, CA, USA).

\section{Cell culture}

NIH 3T3 and COS-7 cells were cultured in Dulbecco's modified Eagle's medium (DMEM) supplemented with 10\% (v/v) fetal bovine serum (FBS), $100 \mathrm{U} / \mathrm{ml}$ penicillin, $100 \mu \mathrm{g} / \mathrm{ml}$ streptomycin, and $25 \mu \mathrm{g} / \mathrm{ml}$ amphotericin B. Human neuroblastoma SH-SY5Y cells stably expressing wild-type human tTG (SH/tTG) were maintained as described previously ${ }^{42}$ in RPMI 1640 medium supplemented with $20 \mathrm{mM}$ glutamine, $5 \%$ fetal clone II serum, $10 \%$ horse serum, $100 \mathrm{U} / \mathrm{ml}$ penicillin, $100 \mu \mathrm{g} / \mathrm{ml}$ streptomycin, $25 \mu \mathrm{g} / \mathrm{ml}$ amphotericin B, and $100 \mu \mathrm{g} / \mathrm{ml} \mathrm{G418} \mathrm{(Geneticin).} \mathrm{Stock} \mathrm{cultures} \mathrm{were}$ maintained at $37^{\circ} \mathrm{C}$ in a humidified atmosphere consisting of $5 \% \mathrm{CO}_{2} / 95 \%$ air. Exposure to calphostin $\mathrm{C}$ was routinely carried out on late log (i.e. $80 \%$ confluent) cultures in the continuous presence of fluorescent light. In some experiments (see Results), cells were preincubated with the transglutaminase inhibitor MDC $(0.5 \mathrm{mM})$ for $1 \mathrm{~h}$ before the addition of calphostin $\mathrm{C}$. The final concentrations of diluent/vehicle (dimethylsulfoxide or methanol) never exceeded $0.1 \%(\mathrm{v} / \mathrm{v})$.

\section{In situ tTG activity assay}

$\mathrm{NIH}$ 3T3 cells were labeled for $2 \mathrm{~h}$ with $1 \mathrm{mM}$ BAP prior to treatment with calphostin C. Cells not exposed to calphostin $C$ served as controls. The cells were subsequently rinsed twice with $\mathrm{PBS}$, harvested in lysis buffer (50 mM Tris- $\mathrm{HCl} \mathrm{pH} \mathrm{7.5,} \mathrm{0.3} \mathrm{M} \mathrm{NaCl,} 5 \mathrm{mM}$ EGTA, $1 \mathrm{mM}$ EDTA, 0.5\% Triton X-100, 0.5\% NP40, $0.1 \mathrm{mM}$ PMSF, $1 \mu \mathrm{g} / \mathrm{ml}$ leupeptin, and $1 \mu \mathrm{g} / \mathrm{ml}$ aprotinin), and sonicated on ice. The protein concentrations in the lysates were determined by the modified Bradford protein assay (Bio-Rad Laboratories, Mississauga, Ontario, Canada). The tTG activity was 
quantified by measuring the transglutaminase-dependent incorporation of BAP into proteins by a microplate assay as described previously ${ }^{43}$.

\section{Plasmid and transfection}

The T7-tagged rat DLK expression vector was a gift of Dr. S-i Hirai (Yokohama City University School of Medicine, Japan). Transient transfections of COS-7 cells seeded at $2.5 \times 10^{5}$ viable cells per 60 $\mathrm{mm}$ dish were carried out by using $1 \mu \mathrm{g}$ of the expression vector and FuGENE 6 transfection reagent (Roche Diagnostics, Laval, Québec, Canada), according to the protocol suggested by the manufacturer. Cells were harvested and processed for immunoblot analyses or in vitro kinase assays $48 \mathrm{~h}$ after transfection.

\section{Construction of recombinant adenoviruses encoding wild-type and kinase-defective DLK}

Recombinant adenoviruses expressing the wild-type (Ad-DLK) and kinase-defective mutant (Ad-DLK[K185R]) forms of DLK were produced according to the method of $\mathrm{He}$ et al. ${ }^{44}$. Briefly, $\mathrm{T} 7$ epitope-tagged wild-type and kinase-defective rat DLK cDNAs were excised from either pSRHisMUK (kindly provided by Dr. S-i Hirai) or pSRHis-MUK[K185R] (a plasmid in which lysine 185 of the ATP-binding domain was replaced by arginine using the Stratagen QuickChange site-directed mutagenesis kit) and directionally subcloned into the shuttle vector pAdTrack-CMV by standard cloning procedures. The resulting shuttle plasmids were electroporated along with the adenoviral backbone vector pAdEasy-1 into Escherichia coli BJ5183 cells. Recombinants adenoviruses were propagated in HEK293 cells, purified by cesium chloride gradient centrifugation, dialyzed, and titered by plaque assay in HEK293 cells. A recombinant adenovirus expressing green fluorescent protein (Ad-GFP) was generated by the same procedure and used as a control in this study.

\section{Adenovirus infection}

Subconfluent SH/tTG cells were infected with the recombinant adenoviruses at a multiplicity of infection (MOI) of 5 in DMEM supplemented with $2 \%$ (v/v) FBS, $0.8 \mathrm{mM} \mathrm{L-arginine,} 100 \mathrm{U} / \mathrm{ml}$ penicillin, $100 \mu \mathrm{g} / \mathrm{ml}$ streptomycin, and $25 \mu \mathrm{g} / \mathrm{ml}$ amphotericin B. After $1 \mathrm{~h}$, the medium was replaced with RPMI-1640 containing $1 \%$ Fetal Clone II serum and $4 \%$ horse serum, and the cells were incubated for an additional $24 \mathrm{~h}$. The cells were then treated with calphostin $C$ for the indicated time points, harvested and processed for further analyses.

\section{Preparation of cell lysates and immunoblotting}

Cells were lysed for $60 \mathrm{~min}$ at $4^{\circ} \mathrm{C}$ in lysis buffer $(15 \mathrm{mM}$ Tris- $\mathrm{HCl} \mathrm{pH} \mathrm{7.4,}$ $1 \%$ Triton $\mathrm{X}-100,0.2 \%$ sodium dodecyl sulfate (SDS), $0.5 \%$ sodium deoxycholate, $150 \mathrm{mM} \mathrm{NaCl}, 1 \mathrm{mM} \mathrm{MgCl}_{2}, 1 \mathrm{mM}$ EGTA, $10 \mathrm{mM} \beta$ mercaptoethanol, $0.2 \mathrm{mM}$ sodium orthovanadate, $1 \mathrm{mM}$ phenylmethylsulfonyl fluoride, $1 \mu \mathrm{g} / \mathrm{ml}$ leupeptin, and $1 \mu \mathrm{g} / \mathrm{ml}$ aprotinin). Lysates were clarified by centrifugation $\left(12000 \times g\right.$ for $10 \mathrm{~min}$ at $4^{\circ} \mathrm{C}$ ) and the concentration of total protein in the supernatant fraction was quantified by the modified Bradford protein assay (Bio-Rad Laboratories, Mississauga, Ontario, Canada). For immunoblotting, equal amounts of proteins were fractionated by SDS-polyacrylamide gel electrophoresis (PAGE) and transferred onto polyvinylidene difluoride (PVDF) membranes (Roche Diagnostics, Laval, Québec, Canada) using a semidry transfer apparatus (Amersham Pharmacia Biotech, Inc., Baie d'Urfé, Québec, Canada).
Membranes were blocked overnight at $4^{\circ} \mathrm{C}$ or for $1 \mathrm{~h}$ at room temperature in $20 \mathrm{mM}$ Tris, pH 7.5, $150 \mathrm{mM} \mathrm{NaCl}, 0.1 \%$ Tween-20 (TBS-T) containing $5 \%$ skim milk powder before addition of the primary antibody ( $1 \mathrm{~h}$ at room temperature or overnight at $4^{\circ} \mathrm{C}$ ). Immunoreactive bands were detected by enhanced chemiluminescence using secondary horseradish peroxidase-conjugated antibodies (ECL Plus Western blotting kit, Amersham Pharmacia Biotech, Inc.).

\section{Immunocomplex kinase assay for DLK}

COS-7 cells transiently transfected with a T7-tagged rat DLK expression vector were incubated in the presence or absence of calphostin $\mathrm{C}$ and homogenized in lysis buffer $(50 \mathrm{mM}$ Tris- $\mathrm{HCl}$ pH 7.4, $1 \%$ Triton X-100, $150 \mathrm{mM} \mathrm{NaCl}, 5 \mathrm{mM}$ EDTA, $0.2 \mathrm{mM}$ sodium orthovanadate, $0.2 \mathrm{mM}$ sodium fluoride, $1 \mathrm{mM}$ phenylmethylsulfonyl fluoride, $1 \mu \mathrm{g} / \mathrm{ml}$ leupeptin, and $1 \mu \mathrm{g} / \mathrm{ml}$ aprotinin). Lysates were clarified by centrifugation and the concentration of total protein in the supernatant fraction was quantified using the modified Bradford protein assay (Bio-Rad Laboratories). Typically, $600 \mu \mathrm{g}$ of protein extract were incubated for $2 \mathrm{~h}$ at $4{ }^{\circ} \mathrm{C}$ with constant rotation using a T7-tag monoclonal antibody (Novagen, $1: 2000$ dilution) and protein A-sepharose beads. After incubation, the immunocomplexes were washed three times with lysis buffer and three times with kinase buffer $\left(10 \mathrm{mM}\right.$ Tris- $\mathrm{HCl} \mathrm{pH} 7.4,150 \mathrm{mM} \mathrm{NaCl}, 10 \mathrm{mM} \mathrm{MgCl}_{2}$, $0.5 \mathrm{mM}$ DTT, $0.1 \mathrm{mM}$ phenylmethylsulfonyl fluoride, $0.2 \mathrm{mM}$ sodium orthovanadate, $1 \mu \mathrm{g} / \mathrm{ml}$ leupeptin, $1 \mu \mathrm{g} / \mathrm{ml}$ aprotinin). Immunocomplex kinase assays were performed by incubating the immune complexes in $40 \mu \mathrm{l}$ of kinase buffer containing $2.5 \mu \mathrm{Ci}$ of $\left[\gamma^{32} \mathrm{P}\right]$ ATP (Amersham Pharmacia Biotech Inc.), $25 \mu \mathrm{M}$ ATP, and $1 \mu \mathrm{g}$ of myelin basic protein as substrate. Following a $20 \mathrm{~min}$ incubation at $30^{\circ} \mathrm{C}$, the reaction was stopped by adding an appropriate volume of $6 \times$ SDS-PAGE sample buffer and boiling for $5 \mathrm{~min}$. Phosphorylated proteins were visualized by autoradiography after fractionation by SDS-PAGE.

\section{JNK assay}

Phosphorylation of endogenous JNK was detected by immunoblotting cell lysates with an antibody specific to phospho- $\left(\mathrm{pThr}^{183} \mathrm{pTyr}{ }^{185}\right)-\mathrm{JNK}$. Alternatively, JNK activity was measured by a solid-state kinase assay in which GST-C-Jun ${ }_{1-79}$ (GST-Jun) bound to glutathione-Sepharose 4B beads was used to affinity purify endogenous JNK from cell lysates as described previously. ${ }^{45}$

\section{Acknowledgements}

We thank Drs. Marco Di Fruscio, Luc Gaudreau, and Alain Lavigueur for critical reading of the manuscript and Drs. S-i Hirai and Nathalie Rivard for pSRHis-MUK and GST-c-Jun constructs, respectively. This work was supported by grants from the Canadian Institutes of Health Research (CIHR) and the Natural Sciences and Engineering Research Council of Canada.

\section{References}

1. Fesus $L$ and Piacentini $M(2002)$ Transglutaminase 2: an enigmatic enzyme with diverse functions. Trends Biochem. Sci. 27: 534-539

2. Griffin M, Casadio R and Bergamini CM (2002) Transglutaminases: nature's biological glues. Biochem. J. 368: 377-396

3. Lorand L and Graham RM (2003) Transglutaminases: crosslinking enzymes with pleiotropic functions. Nat. Rev. Mol. Cell. Biol. 4: 140-156 
4. Lee KN, Birckbichler PJ and Patterson Jr MK (1989) GTP hydrolysis by guinea pig liver transglutaminase. Biochem. Biophys. Res. Commun. 162: 1370-1375

5. Achyuthan KE and Greenberg CS (1987) Identification of a guanosine triphosphate-binding site on guinea pig liver transglutaminase. Role of GTP and calcium ions in modulating activity. J. Biol. Chem. 262: 1901-1906

6. Nakaoka H, Perez DM, Baek KJ, Das T, Husain A, Misono K, Im MJ and Graham RM (1994) Gh: a GTP-binding protein with transglutaminase activity and receptor signaling function. Science 264: 1593-1596

7. Feng JF, Rhee SG and Im MJ (1996) Evidence that phospholipase delta1 is the effector in the Gh (transglutaminase II)-mediated signaling. J. Biol. Chem. 271: $16451-16454$

8. Melino G, Draoui M, Bellincampi L, Bernassola F, Bernardini S, Piacentini M, Reichert U and Cohen P (1997) Retinoic acid receptors alpha and gamma mediate the induction of 'tissue' transglutaminase activity and apoptosis in human neuroblastoma cells. Exp. Cell Res. 235: 55-61

9. Thomazy VA and Davies PJ (1999) Expression of tissue transglutaminase in the developing chicken limb is associated both with apoptosis and endochondral ossification. Cell Death Differ. 6: 146-154

10. Autuori F, Farrace MG, Oliverio S, Piredda L and Piacentini M (1998) Tissue' transglutaminase and apoptosis. Adv. Biochem. Eng. Biotechnol. 62: 129-136

11. Gentile V, Thomazy V, Piacentini M, Fesus L and Davies PJ (1992) Expression of tissue transglutaminase in Balb-C $3 T 3$ fibroblasts: effects on cellular morphology and adhesion. J. Cell Biol. 119: 463-474

12. Melino G, Annicchiarico-Petruzzelli M, Piredda L, Candi E, Gentile V, Davies PJ and Piacentini M (1994) Tissue transglutaminase and apoptosis: sense and antisense transfection studies with human neuroblastoma cells. Mol. Cell. Biol. 14: 6584-6596

13. Tucholski J and Johnson GVW (2002) Tissue transglutaminase differentially modulates apoptosis in a stimuli-dependent manner. J. Neurochem. 81: 780791

14. Oliverio S, Amendola A, Rodolfo C, Spinedi A and Piacentini M (1999) Inhibition of 'tissue' transglutaminase increases cell survival by preventing apoptosis. $J$ Biol Chem. 274: 34123-34128

15. Antonyak MA, Singh US, Lee DA, Boehm JE, Combs C, Zgola MM, Page RL and Cerione RA (2001) Effects of tissue transglutaminase on retinoic acidinduced cellular differentiation and protection against apoptosis. J. Biol. Chem. 276: 33582-33587

16. Antonyak MA, Boehm JE and Cerione RA (2002) Phosphoinositide 3-kinase activity is required for retinoic acid-induced expression and activation of the tissue transglutaminase. J. Biol. Chem. 277: 14712-14716

17. Hickman ES, Moroni MC and Helin K (2002) The role of p53 and pRB in apoptosis and cancer. Curr. Opin. Genet. Dev. 12: 60-66

18. Oliverio S, Amendola A, Di Sano F, Farrace MG, Fesus L, Nemes Z, Piredda L, Spinedi A and Piacentini M (1997) Tissue transglutaminase-dependent posttranslational modification of the retinoblastoma gene product in promonocytic cells undergoing apoptosis. Mol. Cell. Biol. 17: 6040-6048

19. Boehm JE, Singh U, Combs C, Antonyak MA and Cerione RA (2002) Tissue transglutaminase protects against apoptosis by modifying the tumor suppressor protein $\mathrm{p} 110 \mathrm{Rb}$. J. Biol. Chem. 277: 20127-20130

20. Hébert SS, Daviau A, Grondin G, Latreille M, Aubin RA and Blouin R (2000) The mixed lineage kinase DLK is oligomerized by tissue transglutaminase during apoptosis. J. Biol. Chem. 275: 32482-32490

21. Gallo KA and Johnson GL (2002) Mixed-lineage kinase control of JNK and p38 MAPK pathways. Nat. Rev. Mol. Cell. Biol. 3: 663-672

22. Xu Z, Maroney AC, Dobrzanski P, Kukekov NV and Greene LA (2001) The MLK family mediates c-Jun N-terminal kinase activation in neuronal apoptosis. Mol. Cell. Biol. 21: 4713-4724

23. Ip YT and Davis RJ (1998) Signal transduction by the c-Jun N-terminal kinase (JNK) - from inflammation to development. Curr. Opin. Cell Biol. 10: 205-219

24. Davis RJ (2000) Signal transduction by the JNK group of MAP kinases. Cell 103: 239-252

25. Lin A (2003) Activation of the JNK signaling pathway: breaking the brake on apoptosis. BioEssays 25: 17-24
26. Ikemoto H, Tani E, Matsumoto T, Nakano A and Furuyama J (1995) Apoptosis of human glioma cells in response to calphostin $\mathrm{C}$, a specific protein kinase $\mathrm{C}$ inhibitor. J. Neurosurg. 83: 1008-1016

27. Zhu DM, Narla RK, Fang WH, Chia NC and Uckun FM (1998) Calphostin C triggers calcium-dependent apoptosis in human acute lymphoblastic leukemia cells. Clin. Cancer Res. 4: 2967-2976

28. Lu W, Strohecker A and Ou JH (2001) Post-translational modification of the hepatitis $C$ virus core protein by tissue transglutaminase. J. Biol. Chem. 276: 47993-47999

29. Mayne GC and Murray AW (1998) Evidence that protein kinase C epsilon mediates phorbol ester inhibition of calphostin C- and tumor necrosis factoralpha-induced apoptosis in U937 histiocytic lymphoma cells. J. Biol. Chem. 273: 24115-24121

30. Pollack IF and Kawecki S (1997) The effect of calphostin C, a potent photodependent protein kinase $\mathrm{C}$ inhibitor, on the proliferation of glioma cells in vitro. J. Neurooncol. 31: 255-266

31. Zhang H, Koty PP, Mayotte J and Levitt ML (1999) Induction of multiple programmed cell death pathways by IFN-beta in human non-small-cell lung cancer cell lines. Exp. Cell Res. 247: 133-141

32. Lazebnik YA, Kaufmann SH, Desnoyers S, Poirier GG and Earnshaw WC (1994) Cleavage of poly(ADP-ribose) polymerase by a proteinase with properties like ICE. Nature 371: 46-347

33. Nihalani D, Meyer D, Pajni S and Holzman LB (2001) Mixed lineage kinasedependent JNK activation is governed by interactions of scaffold protein JIP with MAPK module components. EMBO J. 20: 3447-3458

34. Chen JS and Mehta K (1999) Tissue transglutaminase: an enzyme with a split personality. Int. J. Biochem. Cell Biol. 31: 817-836

35. Melino $G$ and Piacentini M (1998) Tissue' transglutaminase in cell death: a downstream or a multifunctional upstream effector? FEBS Lett. 430: 59-63

36. Piacentini M, Farrace MG, Mataresse P, Ciccosanti F, Falasca L, Rodolfo C, Giammarioli AM, Verderio E, Griffin M and Malorni W (2002) Transglutaminase overexpression sensitizes neuronal cell lines to apoptosis by increasing mitochondrial membrane potential and cellular oxidative stress. J. Neurochem. 81: 1061-1072

37. Yang DD, Kuan CY, Whitmarsh AJ, Rincon M, Zheng TS, Davis RJ, Rakic $P$ and Flavell RA (1997) Absence of excitotoxicity-induced apoptosis in the hippocampus of mice lacking the Jnk3 gene. Nature 389: 865-870

38. Troy CM, Rabacchi SA, Xu Z, Maroney AC, Connors TJ, Shelanski ML and Greene LA (2001) beta-Amyloid-induced neuronal apoptosis requires c-Jun Nterminal kinase activation. J. Neurochem. 77: 157-164

39. Maroney AC, Finn JP, Bozyczko-Coyne D, O'Kane TM, Neff NT, Tolkovsky AM, Park DS, Yan CY, Troy CM and Greene LA (1999) CEP-1347 (KT7515), an inhibitor of JNK activation, rescues sympathetic neurons and neuronally differentiated PC12 cells from death evoked by three distinct insults. J. Neurochem. 73: 1901-1912

40. Ham J, Babij C, Whitfield J, Pfarr CM, Lallemand D, Yaniv M and Rubin LL (1995) A c-Jun dominant negative mutant protects sympathetic neurons against programmed cell death. Neuron 14: 927-939

41. Douziech M, Laberge G, Grondin G, Daigle N and Blouin R (1999) Localization of the mixed-lineage kinase DLK/MUK/ZPK to the Golgi apparatus in NIH 3T3 cells. J. Histochem. Cytochem. 47: 1287-1296

42. Tucholski J Lesort and Johnson GVW (2001) Tissue transglutaminase is essential for neurite outgrowth in human neuroblastoma SH-SY5Y cells. Neuroscience 102: 481-491

43. Zhang J, Lesort M, Guttmann RP and Johnson GVW (1998) Modulation of the in situ activity of tissue transglutaminase by calcium and GTP. J. Biol. Chem. 273: 2288-2295

44. He TC, Zhou S, da Costa LT, Yu J, Kinzler KW and Vogelstein B (1998) A simplified system for generating recombinant adenoviruses. Proc. Natl. Acad. Sci. USA 95: 2509-2514

45. Hibi M, Lin A, Smeal T, Minden A and Karin M (1993) Identification of an oncoprotein- and UV-responsive protein kinase that binds and potentiates the c-Jun activation domain. Genes Dev. 7: 2135-2148 